\title{
Existence of solutions of a new system of generalized variational inequalities in Banach spaces
}

\author{
Somyot Plubtieng ${ }^{*}$ and Tipphawan Thammathiwat
}

* Correspondence: somyotp@nu.ac. th

Department of Mathematics, Faculty of Science, Naresuan University, Phitsanulok 65000, Thailand

\begin{abstract}
In this article, we consider the solutions of the system of generalized variational inequality problems in Banach spaces. By employing the generalized projection operator, the well-known Fan's KKM theorem and Kakutani-Fan-Glicksberg fixed point theorem, we establish some new existence theorems of solutions for two classes of generalized set-valued variational inequalities in reflexive Banach spaces under some suitable conditions.
\end{abstract}

AMS Subject classification: 47H04; 47H99; 47J20; 47J40.

Keywords: system of generalized variational inequalities, generalized projection, reflexive Banach spaces

\section{Introduction}

Let $E$ be a Banach space, $E^{*}$ be the dual space of $E$, and let $\langle\cdot, \cdot\rangle$ denotes the duality pairing of $E^{*}$ and $E$. If $E$ is a Hilbert space and $K$ is a nonempty, closed and convex subset of $E$, then it is well known that the metric projection operator $P_{K}: E \rightarrow K$ plays an important role in nonlinear functional analysis, optimization theory, fixed point theory, nonlinear programming, game theory, variational inequality problem, and complementarity problems, etc. (see example, [1-32] and the references therein.)

Let $K$ be a nonempty, closed and convex subset of a Hilbert space $H$ and let $A: K \rightarrow$ $H$ be a mapping. The classical variational inequality problem, denoted by $\operatorname{VIP}(A, K)$, is to find $x^{*} \in K$ such that

$$
\left\langle A x^{*}, z-x^{*}\right\rangle \geq 0
$$

for all $z \in K$. The variational inequality has emerged as a fascinating and interesting branch of mathematical and engineering sciences with a wide range of applications in industry, finance, economics, social, ecology, regional, pure, and applied sciences; see, e.g., $[3,10,11,17,21-24,29]$ and the references therein. Related to the variational inequalities, we have the problem of finding the fixed points of the nonexpansive mappings, which is the current interest in functional analysis. It is natural to consider the unified approach to these different problems; see e.g. [17,20,22].

The system of variational inequality problems are the model of several equilibrium problems, namely, traffic equilibrium problem, the spatial equilibrium problem, the

(c) 2012 Plubtieng and Thammathiwat; licensee Springer. This is an Open Access article distributed under the terms of the Creative Commons Attribution License (http://creativecommons.org/licenses/by/2.0), which permits unrestricted use, distribution, and reproduction in any medium, provided the original work is properly cited. 
Nash equilibrium, the general equilibrium programming problem, etc. For further detail see $[2,6,12,13,18,33]$ and the references therein. In $[6,18]$, some solution methods are proposed. However, the existence of a solution of system of variational inequalities is studied in $[2,6,12,13,33]$.

On the other hand, Verma [23-26] introduced and studied a two step model for some systems of variational inequalities which were difference from the sense of Pang [18] and developed some iterative algorithms for approximating the solutions of these systems in Hilbert spaces base on the convergence analysis of a two step projection method. In 2011, Yao et al. [30] extended the main results of Verma [26] from the Hilbert spaces to the Banach spaces.

In 1994, Alber [34] introduced the generalized projection $\pi_{K}: E^{*} \rightarrow K$ and $\Pi_{K}: E \rightarrow$ $K$ from Hilbert spaces to uniformly convex and uniformly smooth Banach spaces and studied their in detail. In [35], Alber presented some applications of the generalized projections to approximately solve variational inequalities (1.1) and von Neumann intersection problem in Banach spaces. Let $A: K \rightarrow E^{* *}$ be a mapping and let us find $x^{*} \in K$ such that

$$
\left\langle A x^{*}-\xi, z-x^{*}\right\rangle \geq 0, \quad \forall z \in K,
$$

where $\xi \in E^{*}$.

Recently, Li [16] extended the generalized projection operator $\pi_{K}: E^{*} \rightarrow K$ from uniformly convex and uniformly smooth Banach spaces to reflexive Banach spaces and studied some properties of generalized projection operator with applications to solve the variational inequality (1.1) in Banach spaces. Very recently, the generalized variational inequality problem $(\operatorname{GVIP}(A, K))$ has been studied by many authors (for example, see $[19,28,36,37])$. It is the problem to find $x^{*} \in K$ such that there exists $u^{*} \in A x^{*}$ satisfying

$$
\left\langle u^{*}, z-x^{*}\right\rangle \geq 0, \quad \forall z \in K .
$$

where $A: K \rightarrow 2^{E^{*}}$ is a multivalued mapping with nonempty values and $2^{E^{*}}$ denotes the family of all subset of $E^{*}$.

In 2009, Wong et al. [27] studied the generalized variational inequality problems defined by a multivalued mapping $T$, a nonempty closed convex subset $K$ of a Banach space $E$ and $b \in E^{*}$ is to find $\bar{x} \in K$ such that there exists $\bar{u} \in T(\bar{x})$ satisfying

$$
\langle\bar{u}-b, y-\bar{x}\rangle \leq 0, \quad \text { for all } y \in K,
$$

in reflexive and smooth Banach spaces by using generalized projection operator, Fan's KKM theorem and minimax theorem.

In this article, we consider the problem for finding the solution of the system of generalized variational inequality problem (1.3) in the sense of Verma [23]. Let $K$ be a nonempty, closed and convex subset of $E$ and $A, B: K \rightarrow 2^{E^{*}}$ be two multivalued mappings with nonempty values, where $2^{E^{*}}$ denotes the family of all subset of $E^{*}$. The system of generalized variational inequality problem (SGVIP(A,B,K)) is to find $\left(x^{*}, y^{*}\right) \in K$ $\times K$ such that there exist $u^{*} \in A y^{*}, v^{*} \in B x^{*}$ satisfying

$$
\left\{\begin{array}{l}
\left\langle u^{*}, z-x^{*}\right\rangle \geq 0, \forall x \in K \\
\left\langle v^{*}, z-y^{*}\right\rangle \geq 0, \forall x \in K
\end{array}\right.
$$


If $A$ and $B$ are single-valued, then the system of generalized variational inequality problem is reduced to find $\left(x^{*}, y^{*}\right) \in K \times K$ such that

$$
\left\{\begin{array}{l}
\left\langle A y^{*}, z-x^{*}\right\rangle \geq 0, \forall z \in K \\
\left\langle B x^{*}, z-y^{*}\right\rangle \geq 0, \forall z \in K
\end{array}\right.
$$

which is called a system of variational inequality problem (SVIP(A,B,K)).

Remark 1.1. (i) $x^{*} \in \operatorname{GVIP}(A, K)$ if and only if $\left(x^{*}, x^{*}\right) \in \operatorname{SGVIP}(A, A, K)$.

(ii) $x^{*} \in \operatorname{VIP}(A, K)$ if and only if $\left(x^{*}, x^{*}\right) \in \operatorname{SVIP}(A, A, K)$.

The purpose of this article is to establish some existence results of solutions for the system variational inequalities (1.3) in reflexive Banach spaces by employing the properties of the generalized projection operator, the well-known Fan's KKM theorem and Kakutani-Fan-Glicksberg theorem.

\section{Preliminaries}

Let $E$ be a real Banach space and let $S=\{x \in E:\|x\|=1\}$ be the unit sphere of $E$. A Banach space $E$ is said to be strictly convex if for any $x, y \in S$,

$$
x \neq y \text { implies }\left\|\frac{x+y}{2}\right\|<1 .
$$

It is also said to be uniformly convex if for each $\varepsilon \in(0,2]$, there exists $\delta>0$ such that for any $x, y \in S$,

$$
\|x-y\| \geq \varepsilon \text { implies }\left\|\frac{x+y}{2}\right\|<1-\delta .
$$

It is known that a uniformly convex Banach space is reflexive and strictly convex; and we define a function $\delta:[0,2] \rightarrow[0,1]$ called the modulus of convexity of $E$ as follows:

$$
\delta(\varepsilon)=\inf \left\{1-\left\|\frac{x+y}{2}\right\|: x, y \in E,\|x\|=\|y\|=1,\|x-y\| \geq \varepsilon\right\} .
$$

Then $E$ is uniformly convex if and only if $\delta(\varepsilon)>0$ for all $\varepsilon \in(0,2]$.

A Banach space $E$ is said to be locally uniformly convex if for each $\varepsilon>0$ and $x \in S$, there exists $\delta(\varepsilon, x)>0$ for $y \in S$,

$$
\|x-y\| \geq \varepsilon \text { implies }\left\|\frac{x+y}{2}\right\|<1-\delta(\varepsilon, x)
$$

From the above definition, it is easy to see that the following implications are valid: $E$ is uniformly convex $\Rightarrow E$ is locally uniformly convex $\Rightarrow E$ is strictly convex

A Banach space $E$ is said to be smooth if the limit

$$
\lim _{t \rightarrow 0} \frac{\|x+t y\|-\|x\|}{t}
$$

exists for all $x, y \in S$. It is also said to be uniformly smooth if the limit (2.5) is attained uniformly for $x, y \in S$. We recall that $E$ is uniformly convex if and only if $E^{*}$ is uniformly smooth. It is well known that $E$ is smooth if and only if $E^{*}$ is strictly convex. The mapping $J: E \rightarrow E^{*}$ defined by

$$
J(x)=\left\{x * \in E:\langle x *, x\rangle=\|x\|^{2}=\|x *\|^{2}\right\}, \quad \text { for all } x \in E,
$$


is called the duality mapping of $E$. It is known that $J(x)=\partial\left(\frac{1}{2}\|x\|^{2}\right)$, where $\partial \varphi(x)$ denotes the subdifferential of $\varphi$ at $x$. The following properties of duality mapping $J$ which are useful for the rest of this work.

Proposition 2.1. [38] Let E be a reflexive Banach space and $E^{*}$ be strictly convex.

(i) The duality mapping $J: E \rightarrow E^{*}$ is single-valued, surjective and bounded.

(ii) If $E$ and $E^{*}$ are locally uniformly convex, then $J$ is a homeomorphism, that is, $J$ and $J^{1}$ are continuous single-valued mappings.

Next, we consider the functional $V: E^{*} \times E \rightarrow \mathbb{R}$ defined as

$$
V(\varphi, x)=\|\varphi\|^{2}-2\langle\varphi, x\rangle+\|x\|^{2}, \text { forall } \varphi \in E * \text {, and } x \in E \text {. }
$$

It is clear that $V(\phi, x)$ is continuous and the map $x \mapsto V(\phi, x)$ and $\phi \mapsto V(\phi, x)$ are convex and $(\|\phi\|-\|x\|)^{2} \leq V(\phi, x) \leq(\|\phi\|+\|x\|)^{2}$. We remark that the main Lyapunov functional $V$ was first introduced by Alber [35] and its properties were studied there. By using this functional, Alber defined a generalized projection operator on uniformly convex and uniformly smooth Banach spaces which was further extended by Li [16] on reflexive Banach spaces.

Definition 2.2. [16] Let $E$ be reflexive Banach space with its dual $E^{*}$ and $K$ be a nonempty, closed and convex subset of $E$. The operator $\pi_{K}: E^{*} \rightarrow K$ defined by

$$
\pi_{K}(\varphi)=\left\{x \in K: V(\varphi, x)=\inf _{y \in K} V(\varphi, y)\right\}, \quad \text { for all } \varphi \in E *,
$$

is said to be a generalized projection operator. For each $\phi \in E^{*}$, the set $\pi_{K}(\phi)$ is called the generalized projection of $\phi$ on $K$.

We mention the following useful properties of the operator $\pi_{k}(\phi)$.

Lemma 2.3. [16] Let $E$ be a reflexive Banach space with its dual $E^{*}$ and $K$ be a nonempty closed convex subset of $E$, then the following properties hold:

(i) The operator $\pi_{K}: E^{*} \rightarrow 2^{K}$ is single-valued if and only if $E$ is strictly convex.

(ii) If $E$ is smooth, then for any given $\phi \in E^{*}, x \in \pi_{K} \phi$ if and only if $\langle\phi-J(x), x-y\rangle$ $\geq 0, \forall y \in K$.

(iii) If $E$ is strictly convex, then the generalized projection operator $\pi_{K}: E^{*} \rightarrow K$ is continuous.

Lemma 2.4. [5] In every reflexive Banach space, an equivalent norm can be introduced so that $E$ and $E^{*}$ are locally uniformly convex and thus also strictly convex with respect to the new norm on $E$ and $E^{*}$.

From Lemma 2.4, we can assume for the rest of this work that the norm $\|\cdot\|$ of the reflexive Banach space $E$ is such that $E$ and $E^{*}$ are locally uniformly convex. In this case, we note that the generalized metric projection operator $\pi_{K}$ and the duality mapping $J$ are single-valued and continuous.

Lemma 2.5. [38] Let $A$ and $B$ be convex subsets of some real topological vector space with $B$ is compact and let $p: A \times B \rightarrow \mathbb{R}$. If $p(\cdot, b)$ is lower semicontinuous and quasiconvex on $A$ for all $b \in B$ and $p(a, \cdot)$ is upper semicontinuous and quasiconcave on $B$ for all $a \in A$, then 


$$
\inf _{a \in A} \max _{b \in B} p(a, b)=\max _{b \in B} \inf _{a \in A} p(a, b)
$$

Definition 2.6 (KKM mapping). Let $K$ be a nonempty subset of a linear space $E$. A set-valued mapping $G: K \rightarrow 2^{E}$ is said to be a $K K M$ mapping if for any finite subset $\left\{y_{1}, y_{2}, \ldots, y_{n}\right\}$ of $K$, we have

$$
\operatorname{co}\left\{y_{1}, y_{2}, \ldots, y_{n}\right\} \subseteq \bigcup_{i=1}^{n} G\left(y_{i}\right)
$$

where $\operatorname{co}\left\{y_{1}, y_{2}, \ldots, y_{n}\right\}$ denotes the convex hull of $\left\{y_{1}, y_{2}, \ldots, y_{n}\right\}$.

Lemma 2.7 (FanKKM Theorem). Let $K$ be a nonempty convex subset of a Hausdorff topological vector space $E$ and let $G: K \rightarrow 2^{E}$ be a KKM mapping with closed values. If there exists a point $y_{0} \in K$ such that $G\left(y_{0}\right)$ is a compact subset of $K$, then $\cap_{y \in K} G(\gamma) \neq \emptyset$.

Lemma 2.8. [9] Let $K$ be a nonempty compact subset of a locally convex Hausdorff vector topology space E. If $S: K \rightarrow 2^{K}$ is upper semicontinuous and for any $x \in K, S(x)$ is nonempty, convex and closed, then there exists an $x^{*} \in K$ such that $x^{*} \in S\left(x^{*}\right)$.

Lemma 2.9. [39] Let $X$ and $Y$ be two Hausdorff topological vector spaces and $T: X \rightarrow$ $2^{Y}$ be a set-valued mapping. Then the following properties hold:

(i) If $T$ is closed and $\overline{T(X)}$ is compact, then $T$ is upper semicontinuous, where $T(X)=$ $\mathrm{U}_{x \in X} T(x)$ and $\overline{T(X)}$ denotes the closure of the set $T(X)$.

(ii) If $T$ is upper semicontinuous and for any $x \in X, T(x)$ is closed, then $T$ is closed.

(iii) $T$ is lower semicontinuous at $x \in X$ if and only if for any $y \in T(x)$ and any net $\left\{x_{\alpha}\right\}, x_{\alpha} \rightarrow x$, there exists a net $\left\{y_{\alpha}\right\}$ such that $y_{\alpha} \in T\left(x_{\alpha}\right)$ and $y_{\alpha} \rightarrow y$.

\section{Main result}

Proposition 3.1. Let $E$ be a reflexive and smooth Banach space and let $K$ be a closed convex subset of E. Assume that $\alpha, \beta>0$ and $A: K \rightarrow 2^{E^{*}}, B: K \rightarrow 2^{E^{*}}$ are two multivalued mappings with nonempty values. Then $\left(x^{*}, y^{*}\right)$ is a solution of (1.3) if and only if there exist $u^{*} \in A y^{*}, v^{*} \in B x *$ such that

$$
\left\{\begin{array}{l}
x^{*}=\pi_{K}\left[J\left(x^{*}\right)-\alpha u^{*}\right], \\
y^{*}=\pi_{K}\left[J\left(y^{*}\right)-\beta v^{*}\right] .
\end{array}\right.
$$

Proof. It follows from the definition of $\operatorname{SGVIP}(\mathrm{A}, \mathrm{B}, \mathrm{K})$ and Lemma 2.3, that $\left(x^{*}, y^{*}\right)$ is a solution of $(1.3) \Leftrightarrow \exists u^{*} \in A y^{*}, v^{*} \in B x^{*}$ such that

$$
\begin{aligned}
& \left\{\begin{array}{l}
\left\langle u^{*}, z-x^{*}\right\rangle \geq 0, \quad \forall z \in K, \\
\left\langle v^{*}, z-y^{*}\right\rangle \geq 0, \quad \forall z \in K .
\end{array}\right. \\
\Leftrightarrow & \left\{\begin{array}{l}
\left\langle\alpha u^{*}, z-x^{*}\right\rangle \geq 0, \quad \forall z \in K, \\
\left\langle\beta v^{*}, z-y^{*}\right\rangle \geq 0, \quad \forall z \in K .
\end{array}\right. \\
\Leftrightarrow & \left\{\begin{array}{l}
\left\langle J\left(x^{*}\right)-\alpha u^{*}-J\left(x^{*}\right), x^{*}-z\right\rangle \geq 0, \forall z \in K, \\
\left\langle J\left(y^{*}\right)-\beta v^{*}-J\left(y^{*}\right), \gamma^{*}-z\right\rangle \geq 0, \forall z \in K .
\end{array}\right. \\
\Leftrightarrow & \left\{\begin{array}{l}
x^{*}=\pi_{K}\left[J\left(x^{*}\right)-\alpha u^{*}\right], \\
y^{*}=\pi_{K}\left[J\left(y^{*}\right)-\beta v^{*}\right] .
\end{array}\right.
\end{aligned}
$$

Theorem 3.2. Let $E$ be a reflexive and smooth Banach space such that $E$ and $E^{*}$ are locally uniformly convex. Let $K$ be a compact convex subset of $E$. Let $A: K \rightarrow 2^{E^{*}}$ and $B$ $: K \rightarrow 2^{E^{*}}$ be two upper semicontinuous multivalued mappings with nonempty values 
such that $A(x)$ and $B(x)$ are weak* compact and convex for each $x \in K$. Then the problem (1.3) has a solution and the set of solutions (1.3) is closed.

Proof. Step 1. Let $\alpha, \beta>0$ and fixed $x \in K$, for each $z \in K$, the sets $G_{x}(z)$ and $H_{x}(z)$ define as follow

$$
\left\{\begin{array}{l}
G_{x}(z):\left\{y \in K: \inf _{u \in A x}\left(\langle J(y)-\alpha u, 2(z-y)\rangle+\|y\|^{2}\right) \leq\|z\|^{2}\right\} \\
H_{x}(z):\left\{y \in K: \inf _{v \in B x}\left(\langle J(y)-\beta v, 2(z-\gamma)\rangle+\|y\|^{2}\right) \leq\|z\|^{2}\right\} .
\end{array}\right.
$$

(a1) For each $z \in K$, we have $z \in G_{x}(z)$ and $z \in H_{x}(z)$. Hence $G_{x}(z)$ and $H_{x}(z)$ are nonempty subsets of $K$.

(a2) For any finite set $\left\{z_{1}, z_{2}, \ldots, z_{n}\right\} \subset K$ we claim that $\operatorname{co}\left\{z_{1}, z_{2}, \ldots, z_{n}\right\} \subset \bigcup_{j=1}^{n} G_{x}\left(z_{j}\right)$ and $\operatorname{co}\left\{z_{1}, z_{2}, \ldots, z_{n}\right\} \subset \bigcup_{j=1}^{n} H_{x}\left(z_{j}\right)$.

Let $z \in \operatorname{co}\left\{z_{1}, z_{2}, \ldots, z_{n}\right\}$. Then $z=\sum_{j=1}^{n} \lambda_{j} z_{j}$ where $\lambda_{j} \in[0,1]$ and $\sum_{j=1}^{n} \lambda_{j}=1$. We observe that

$$
\sum_{j=1}^{n} \inf _{u \in A x}\left\langle J(z)-\alpha u, 2 \lambda_{j}\left(z_{j}-z\right)\right\rangle \leq \inf _{u \in A x}\left\langle J(z)-\alpha u, 2 \sum_{j=1}^{n} \lambda_{j}\left(z_{j}-z\right)\right\rangle=0 .
$$

Thus,

$$
\sum_{j=1}^{n} \inf _{u \in A x}\left(\left\langle J(z)-\alpha u, 2 \lambda_{j}\left(z_{j}-z\right)\right\rangle+\lambda_{j}\|z\|^{2}\right) \leq\|z\|^{2} \leq \sum_{j=1}^{n} \lambda_{j}\left\|z_{j}\right\|^{2} .
$$

This implies that

$$
\sum_{j=1}^{n} \inf _{u \in A x}\left(\left\langle J(z)-\alpha u, 2 \lambda_{j}\left(z_{j}-z\right)\right\rangle+\lambda_{j}\|z\|^{2}-\lambda_{j}\left\|z_{j}\right\|^{2}\right) \leq 0 .
$$

So there exists $j>0$ such that

$$
\inf _{u \in A x}\left(\left\langle J(z)-\alpha u, 2 \lambda_{j}\left(z_{j}-z\right)\right\rangle+\lambda_{j}\|z\|^{2}-\lambda_{j}\left\|z_{j}\right\|^{2}\right) \leq 0 .
$$

Hence,

$$
\inf _{u \in A x}\left(\left\langle J(z)-\alpha u, 2\left(z_{j}-z\right)\right\rangle+\|z\|^{2}\right) \leq\left\|z_{j}\right\|^{2} .
$$

Therefore $z \in G_{x}\left(z_{j}\right) \subset \bigcup_{j=1}^{n} G_{x}\left(z_{j}\right)$. Similarly, we obtain that there exists $k>0$ such that $z \in H_{x}\left(z_{k}\right) \subset \bigcup_{j=1}^{n} H_{x}\left(z_{j}\right)$. Hence we have the claim. This implies that $G_{x}(\cdot)$ and $H_{x}(\cdot)$ are KKM-mappings.

Step 2. Show that $G_{x}(z)$ and $H_{x}(z)$ are closed for all $z \in K$.

Let $\left\{x_{n}\right\}$ be a sequence in $G_{x}(z)$ such that $x_{n} \rightarrow x_{0}$ in a norm topology. Then there exists $u_{n} \in A x$ such that

$$
\left\langle J\left(x_{n}\right)-\alpha u_{n}, 2\left(z-x_{n}\right)\right\rangle+\left\|x_{n}\right\|^{2}=\inf _{u \in A x}\left(\left\langle J\left(x_{n}\right)-\alpha u, 2\left(z-x_{n}\right)\right\rangle+\left\|x_{n}\right\|^{2}\right) \leq\|z\|^{2} .
$$

Since $A(x)$ is compact, there exists a subsequence $\left\{u_{n_{j}}\right\}$ of $\left\{u_{n}\right\}$ such that $u_{n_{j}} \rightarrow u_{0} \in A(x)$. Thus without loss of generality, we may assume that $u_{n} \rightarrow u_{0}$ and observe that 


$$
\left\langle J\left(x_{n}\right)-\alpha u_{n}, 2\left(z-x_{n}\right)\right\rangle+\left\|x_{n}\right\|^{2} \rightarrow\left\langle J\left(x_{0}\right)-\alpha u_{0}, 2\left(z-x_{0}\right)\right\rangle+\left\|x_{0}\right\|^{2} .
$$

Therefore

$$
\left.\inf _{u \in A x}\left\langle J\left(x_{0}\right)-\alpha u, 2\left(z-x_{0}\right)\right\rangle+\left\|x_{0}\right\|^{2}\right) \leq\left\langle J\left(x_{0}\right)-\alpha u_{0}, 2\left(z-x_{0}\right)\right\rangle+\left\|x_{0}\right\|^{2} \leq\|z\|^{2} .
$$

This implies that $x_{0} \in G_{x}(z)$ and so $G_{x}(z)$ is closed for all $z \in K$. Similarly, we obtain that $H_{x}(z)$ is closed for all $z \in K$. Then $\cap_{z \in K_{K}} G_{y}(z)$ and $\cap_{z \in{ }_{K}} H_{x}(z)$ are also closed.

Step 3. Show that $\bigcap_{z \in K} G_{x}(z) \neq \emptyset \neq \bigcap_{z \in K} H_{z}(z)$.

Since $G_{x}(z)$ and $H_{x}(z)$ are closed subsets of $K$ and $K$ is compact, $G_{x}(z)$ and $H_{x}(z)$ are compact subsets of $K$. It follows from Steps 1, 2, and Lemma 2.7 that $\bigcap_{z \in K} G_{x}(z) \neq \emptyset \neq \bigcap_{z \in K} H_{z}(z)$.

Step 4. Show that the problem (1.3) has a solution.

For any $x, y \in K$, we may choose $\bar{x} \in \bigcap_{z \in K} G_{y}(z)$ and $\bar{y} \in \bigcap_{z \in K} H_{x}(z)$ by Step 3. We define the set-valued mapping $S: K \times K \rightarrow 2^{K \times K}$ by

$$
S(x, y)=(\{\bar{x}\},\{\bar{y}\}) \text { where } \bar{x} \in \bigcap_{z \in K} G_{y}(z) \text { and } \bar{y} \in \bigcap_{z \in K} H_{x}(z), \quad \forall(x, y) \in K \times K \text {. }
$$

By Definition of $S(x, y)$ and Step 3, we obtain that $S(x, y)$ is a nonempty closed convex subset of $K \times K$ for all $(x, y) \in K \times K$. Since $\bigcap_{z \in K} G_{y}(z), \bigcap_{z \in K} H_{x}(z) \subset K$ and $K$ is compact, $\overline{\bigcap_{z \in K} G_{y}(z)}$ and $\overline{\bigcap_{z \in K} H_{y}(z)}$ are compact. We only show that $S$ is a closed mapping. Indeed, let $\left\{\left(x_{n}, y_{n}\right)\right\}$ be a net in $K \times K$ such that $\left(x_{n}, y_{n}\right) \rightarrow\left(x_{0}, y_{0}\right)$ in the norm topology and let $\left(u_{n}, v_{n}\right) \in S\left(x_{n}, y_{n}\right)$ such that $\left(u_{n}, v_{n}\right) \rightarrow\left(u_{0}, v_{0}\right)$. By definition of a mapping $S$, we have $\left(u_{n}, v_{n}\right) \in\left(\left\{\bar{x}_{n}\right\},\left\{\bar{y}_{n}\right\}\right)$ where $\bar{x}_{n} \in \bigcap_{z \in K} G_{y_{n}}(z)$ and $\bar{y}_{n} \in \bigcap_{z \in K} H_{x_{n}}(z)$. That is for each $z \in K, u_{n}=\bar{x}_{n} \in G_{y_{n}}(z)$ and $v_{n}=\bar{y}_{n} \in H_{x_{n}}(z)$. It follows from (3.2) that there exist $a_{n} \in A y_{n}$ and $b_{n} \in B x_{n}$ such that

$$
\left\{\begin{array}{c}
\left\langle J\left(u_{n}\right)-\alpha a_{n}, 2\left(z-u_{n}\right)\right\rangle+\left\|u_{n}\right\|^{2}=\inf _{u \in A y_{n}}\left(\left\langle J\left(u_{n}\right)-\alpha u, 2\left(z-u_{n}\right)\right\rangle+\left\|u_{n}\right\|^{2}\right) \leq\|z\|^{2} \\
\left\langle J\left(v_{n}\right)-\beta b_{n}, 2\left(z-v_{n}\right)\right\rangle+\left\|v_{n}\right\|^{2}=\inf _{v \in B x_{n}}\left(\left\langle J\left(v_{n}\right)-\beta v, 2\left(z-v_{n}\right)\right\rangle+\left\|v_{n}\right\|^{2}\right) \leq\|z\|^{2} .
\end{array}\right.
$$

Now, we define two sets $T_{1}:=\left\{x_{1}, x_{2}, \ldots, x_{n}, \ldots\right\} \cup\left\{x_{0}\right\}$ and

$T_{2}:=\left\{y_{1}, y_{2}, \ldots, y_{n}, \ldots\right\} \cup\left\{y_{0}\right\}$.

It follows from our assumption that $A\left(T_{2}\right)$ and $B\left(T_{1}\right)$ are compact. Thus there exist two subsequences $\left\{a_{n_{j}}\right\}$ of $\left\{a_{n}\right\}$ and $\left\{b_{n_{k}}\right\}$ of $\left\{b_{n}\right\}$ such that $a_{n_{j}} \rightarrow a_{0} \in A\left(T_{2}\right)$ and $b_{n_{k}} \rightarrow b_{0} \in B\left(T_{2}\right)$. Since $A$ and $B$ are upper semicontinuous, $a_{0} \in A y_{0}$ and $b_{0} \in B x_{0}$. Taking $j, k \rightarrow \infty$ in (3.7), we obtain that

$$
\left\{\begin{array}{c}
\inf _{u \in A y_{0}}\left(\left\langle J\left(u_{0}\right)-\alpha u, 2\left(z-u_{0}\right)\right\rangle+\left\|u_{0}\right\|^{2}\right) \leq\left\langle J\left(u_{0}\right)-\alpha a_{0}, 2\left(z-u_{0}\right)\right\rangle+\left\|u_{0}\right\|^{2} \leq\|z\|^{2} \\
\inf _{u \in B x_{0}}\left(\left\langle J\left(v_{0}\right)-\beta v, 2\left(z-v_{0}\right)\right\rangle+\left\|v_{0}\right\|^{2}\right) \\
\leq\left\langle J\left(v_{0}\right)-\beta b_{0}, 2\left(z-v_{0}\right)\right\rangle+\left\|v_{0}\right\|^{2} \leq\|z\|^{2} .
\end{array}\right.
$$

Hence $u_{0} \in G_{y 0}(z)$ and $v_{0} \in H_{x_{0}}(z)$ for all $z \in K$. This implies that $\left(u_{0}, v_{0}\right) \in\left(\left\{u_{0}\right\}\right.$, $\left.\left\{v_{0}\right\}\right)=S\left(x_{0}, y_{0}\right)$. Thus, $S$ is a closed mapping. It follows from Lemma 2.9 that $S$ is upper semicontinuous. By Lemma 2.8, there exists a point $\left(x^{*}, y^{*}\right) \in S\left(x^{*}, y^{*}\right)=(\{\bar{x}\},\{\bar{y}\})$ where $\bar{x} \in \bigcap_{z \in K} G_{y^{*}}(z)$ and $\bar{y} \in \bigcap_{z \in K} H_{x^{*}}(z)$. That is $x^{*}=\bar{x} \in G_{\gamma^{*}}(z)$ and $y^{*}=\bar{y} \in H_{x^{*}}(z)$ for all $z \in K$. By definition of $G_{y^{*}}(z)$ and $H_{x^{* *}}(z)$, we get

$$
\left\{\begin{array}{l}
\inf _{u \in A y^{*}}\left(\left\langle J\left(x^{*}\right)-\alpha u, 2\left(z-x^{*}\right)\right\rangle+\|x\|^{2}\right) \leq\|z\|^{2}, \forall z \in K \\
\inf _{v \in B x^{*}}\left(\left\langle J\left(\gamma^{*}\right)-\beta v, 2\left(z-y^{*}\right)\right\rangle+\|y\|^{2}\right) \leq\|z\|^{2}, \forall z \in K .
\end{array}\right.
$$


This implies that

$$
\left\{\begin{array}{c}
\sup _{z \in K} \inf _{u \in A y^{*}}\left(\left\langle J\left(x^{*}\right)-\alpha u, 2\left(z-x^{*}\right)\right\rangle+\|x\|^{2}-\|z\|^{2}\right) \leq 0 \\
\sup _{z \in K^{\prime n f}} \inf _{v \in B x^{*}}\left(\left\langle J\left(\gamma^{*}\right)-\beta v, 2\left(z-y^{*}\right)\right\rangle+\left\|y^{*}\right\|^{2}-\|z\|^{2}\right) \leq 0
\end{array}\right.
$$

Put

$$
\left\{\begin{array}{l}
p_{1}(u, z)=\left\langle J\left(x^{*}\right)-\alpha u, 2\left(z-x^{*}\right)\right\rangle+\left\|x^{*}\right\|^{2}-\|z\|^{2} \\
p_{2}(v, z)=\left\langle J\left(y^{*}\right)-\beta v, 2\left(z-y^{*}\right)\right\rangle+\left\|y^{*}\right\|^{2}-\|z\|^{2}
\end{array}\right.
$$

Then the functional $p_{1}(\cdot, z), p_{2}(\cdot, z)$ are lower semicontinuous and convex. Also the function $p_{1}(u, \cdot), p_{2}(v, \cdot)$ are upper semicontinuous and concave. Apply minimax theorem, we have

$$
\left\{\begin{array}{l}
\sup _{z \in K} \inf _{u \in A y^{*}} p_{1}(u, z)=\inf _{u, \in A y^{*}} \sup _{z \in K} p_{1}(u, z) \leq 0 \\
\sup _{z \in K} \inf _{v \in B x^{*}} p_{2}(v, z)=\inf _{v, \in B x^{*}} \sup _{z \in K} p_{2}(v, z) \leq 0
\end{array}\right.
$$

Since the functional $u \mapsto \sup _{z \in K} p_{1}(u, z)$ and $v \mapsto \sup _{z \in K} p_{2}(v, z)$ are lower semicontinuous and $A\left(y^{*}\right), B\left(x^{*}\right)$ are compact, there exist $u^{*} \in A\left(y^{*}\right)$ and $v^{*} \in B\left(x^{*}\right)$ such that

$$
\left\{\begin{array}{l}
\sup _{z \in K} p_{1}\left(u^{*}, z\right)=\inf _{u \in A \gamma^{*}} \sup _{z \in K} p_{1}(u, z) \leq 0 \\
\sup _{z \in K} p_{2}\left(v^{*}, z\right)=\inf _{v \in B x^{*}} \sup _{z \in K} p_{2}(v, z) \leq 0
\end{array}\right.
$$

This implies that

$$
\left\{\begin{array}{l}
p_{1}\left(u^{*}, z\right) \leq 0, \forall z \in K \\
p_{2}\left(v^{*}, z\right) \leq 0, \forall z \in K
\end{array}\right.
$$

That is

$$
\left\{\begin{array}{l}
\left\langle J\left(x^{*}\right)-\alpha u^{*}, 2\left(z-x^{*}\right)\right\rangle+\left\|x^{*}\right\|^{2}-\|z\|^{2} \leq 0, \forall z \in K, \\
\left\langle J\left(y^{*}\right)-\beta v^{*}, 2\left(z-y^{*}\right)\right\rangle+\left\|y^{*}\right\|^{2}-\|z\|^{2} \leq 0, \forall z \in K .
\end{array}\right.
$$

So, we obtain that

$$
\left\{\begin{array}{l}
V\left(J\left(x^{*}\right)-\alpha u^{*}, x^{*}\right) \leq V\left(J\left(x^{*}\right)-\alpha u^{*}, z\right), \forall z \in K \\
V\left(J\left(y^{*}\right)-\beta v^{*}, \gamma^{*}\right) \leq V\left(J\left(\gamma^{*}\right)-\beta v^{*}, z\right), \forall z \in K .
\end{array}\right.
$$

By definition of generalized projection operator, we get $x^{*}=\pi_{K}\left(J\left(x^{*}\right)-\alpha u^{*}\right)$ and $y^{*}=$ $\pi_{K}\left(J\left(y^{*}\right)-\beta v^{*}\right)$. It follows from Proposition 3.1 that $\left(x^{*}, y^{*}\right)$ is the solutions of problem (1.3).

Step 5. Show that the set of solutions (1.3) is closed.

Put $T:=\{(x, y) \in K \times K:(x, y)$ is a solution of (1.3) $\}$. Let $\left\{\left(x_{n}, y_{n}\right)\right\}$ be a net in $T$ such that $\left(x_{n}, y_{n}\right) \rightarrow\left(x_{0}, y_{0}\right)$ in the norm topology. By definition (1.3) we obtain that there exist $u_{n} \in A\left(y_{n}\right)$ and $v_{n} \in B\left(x_{n}\right)$ such that

$$
\left\{\begin{array}{l}
\left\langle u_{n}, z-x_{n}\right\rangle \geq 0, \forall z \in K, \\
\left\langle v_{n}, z-y_{n}\right\rangle \geq 0, \forall z \in K .
\end{array}\right.
$$

We define two sets $T_{1}:=\left\{x_{1}, x_{2}, \ldots, x_{n}, \ldots\right\} \cup\left\{x_{0}\right\}$ and $T_{2}:=\left\{y_{1}, y_{2}, \ldots, y_{n}, \ldots\right\} \cup\left\{y_{0}\right\}$.

It follows from our assumption that $A\left(T_{2}\right)$ and $B\left(T_{1}\right)$ are compact. Thus there exist two subsequences $\left\{u_{n_{j}}\right\}$ of $\left\{u_{n}\right\}$ and $\left\{v_{n_{k}}\right\}$ of $\left\{v_{n}\right\}$ such that $u_{n_{j}} \rightarrow u_{0} \in A\left(T_{2}\right)$ and $v_{n_{k}} \rightarrow v_{0} \in B\left(T_{2}\right)$. Since $A$ and $B$ are upper semicontinuous, $u_{0} \in A y_{0}$ and $v_{0} \in B x_{0}$. 
Taking $j, k \rightarrow \infty$ in (3.16), we obtain that

$$
\left\{\begin{array}{l}
\left\langle u_{0}, z-x_{0}\right\rangle \geq 0, \forall z \in K \\
\left\langle v_{0}, z-y_{0}\right\rangle \geq 0, \forall z \in K
\end{array}\right.
$$

Thus $\left(x_{0}, y_{0}\right) \in T$ and so $T$ is closed. This completes the proof.

If $A$ and $B$ are two single-valued mappings, then from Theorem 3.2, we derive the following result.

Corollary 3.3. Let $E$ be a reflexive and smooth Banach space such that $E$ and $E^{*}$ are locally uniformly convex. Let $K$ be a compact convex subset of $E$. Let $A: K \rightarrow E^{*}$ and $B$ : $K \rightarrow E^{*}$ be two continuous single-valued mappings. Then the problem (1.4) has a solution and the set of solutions (1.4) is closed.

\section{Acknowledgements}

The authors would like to thanks The Thailand Research Fund for financial support. Moreover, $T$ is also supported by the Royal Golden Jubilee Program under Grant PHD/0117/2549, Thailand.

\section{Authors' contributions}

This work was carried out in collaboration between all authors. SP gave the ideas of the problems in this research and interpreted the results. $\Pi$ proved the theorems, interpreted the results and wrote the article. All authors defined the research theme, read and approved the manuscript.

\section{Competing interests}

The authors declare that they have no competing interests.

Received: 30 July 2011 Accepted: 16 January 2012 Published: 16 January 2012

\section{References}

1. Baiocchi, C, Capelo, A: Variational and Quasi-Variational Inequalietis, Application to Free Boundary Problem. Wiley, New York/London (1984)

2. Bianchi, M: Pseudo P-monotone operators and variational inequalities. Report 6 Istituto di econometria e Matematica per le decisioni economiche. Universita Cattolica del Sacro Cuore, Milan, Italy (1993)

3. Chang, SS, Lee Joseph, HW, Chan, CK: A new method for solving equilibrium problem fixed point problem and variational inequality problem with application to optimization. Nonlinear Anal. 70, 3307-3319 (2009). doi:10.1016/j. na.2008.04.035

4. Cho, YJ, Fang, YP, Huang, NJ, Hwang, HJ: Algorithms for systems of nonlinear variational inequalities. J Korean Math Soc. 41, 489-499 (2004)

5. Cioranescu, I: Geometry of Banach Spaces Duality Mappings and Nonlinear Problem. Kluwer Acadimic Publishers, Dordrecht (1990)

6. Cohen, G, Chaplais, F: Nested monotony for variational inequalities over a product of spaces and convergence of iterative algorithms. J Optim Theory Appl. 59, 360-390 (1988)

7. Fan, K: A generalization of Tychonoff's fixed point theorem. Math Ann. 142, 305-310 (1961). doi:10.1007/BF01353421

8. Giannessi, F, Maugeri, A: Vairational Inequalities and Network Equilibrium Problems. Plenum, New York (1995)

9. Holmes, RB: Geometric Functional Analysis and its Application. Springer, New York (1975)

10. Isac, G: Complementarity problems. In Lecture Notes in Math, vol. 1528,Springer, Berlin (1992)

11. Isac, G, Sehgal, VM, Singh, SP: An altenate version of a variational inequality. Indian J Math. 41, 25-31 (1999)

12. Kassay, G, Kolumbán, J: System of multi-valued variational variational inequalities. Publ Math Debrecen. 54, 267-279 (1999)

13. Kassay, G, Kolumbán, J, Páles, Z: Factorization of Minty and Stampacchia variational inequality system. European J Oper Res. 143, 377-389 (2002). doi:10.1016/S0377-2217(02)00290-4

14. Kim, JK, Kim, DS: A new system of generalized nonlinear mixed variational inequalities in Hilbert spaces. J Convex Anal. 11, 235-243 (2004)

15. Li, JL: On the existence of solutions of variational inequalities in Banach space. J Math Anal Appl. 295, 115-126 (2004) doi:10.1016/j.jmaa.2004.03.010

16. Li, JL: The generalized projection operator on reflexive Banach spaces and its application. J Math Anal Appl. 306, 55-71 (2005). doi:10.1016/j.jmaa.2004.11.007

17. Nadezhkina, N, Takahashi, W: Weak convergence theorem by an extragradient method for nonexpansive mappings and monotone mappings. J Optim Theory Appl. 128, 191-201 (2006). doi:10.1007/s10957-005-7564-z

18. Pang, JS: Asymmetric variational inequality problems over product sets: Applications and iterative methods. Math Program. 31, 206-219 (1985). doi:10.1007/BF02591749

19. Shih, M-H, Tan, K-K: Browder-Hartman-Stempacchia variational inequalities for multi-valued monotone operators. J Math Anal Appl. 134, 431-440 (1988). doi:10.1016/0022-247X(88)90033-9

20. Suzuki, T: Strong convergence of krasnoselskii and mann's type sequences for one-parameter nonexpansive semigroups without bochner integrals. J Math Anal Appl. 305, 227-239 (2005). doi:10.1016/j.jmaa.2004.11.017 
21. Takahashi, S, Takahashi, W: Viscosity approximation methods for equilibrium problems and fixed point problems in Hilbert spaces. J Math Anal Appl. 311(1):506-515 (2007)

22. Takahashi, W, Toyoda, M: Weak convergence theorems for nonexpansive mappings and monotone mappings. J Optim Theory Appl. 118, 417-428 (2003). doi:10.1023/A:1025407607560

23. Verma, RU: On a new system of nonlinear variational inequalities and associated iterative algorithms. Math Sci Res HotLine. 3(8):65-68 (1999)

24. Verma, RU: Iterative algorithms and a new system of nonlinear quasivariational inequalities. Adv Nonlinear Var Inequal. 4(1):117-124 (2001)

25. Verma, RU: Projection methods, algorithms, and a new system of nonlinear variational inequalities. Comp Math Appl. 41, 1025-1031 (2001). doi:10.1016/50898-1221(00)00336-9

26. Verma, RU: General convergence analysis for two-step projection methods and application to variational problems. Appl Math Lett. 18, 1286-1292 (2005). doi:10.1016/j.aml.2005.02.026

27. Wong, M-M, Ansari, QH, Yao, J-C: Existence of solutions of generalized variational inequalities in reflexive Banach spaces. Appl Math Lett. 22, 197-201 (2009). doi:10.1016/j.aml.2008.03.009

28. Yao, JC: Muti-valued variational inequalities with K-psuedomonotone opeerators. J Optim Theory Appl. 83(2):391-403 (1994). doi:10.1007/BF02190064

29. Yao, JC, Chadli, O: Pseudomonotone Complementarity Problems and variational Inequalities. In: Crouzeix, JP, Haddjissas, N, Schaible, S (eds.) Handbook of Generalized Convexity and Monotonicity. pp. 501-558. Springer, New York (2005)

30. Yao, Y, Liou, Y-C, Kang, SM: Two step projdection methods for a system of variational inequality problems in Banach spaces. J Glob Optim. (2011)

31. Yao, Y, Shahzad, N: Strong convergence of a proximal point algorithm with general errors. Optim Lett. (2011)

32. Yuan, GXZ: KKM Theory and Applications in Nonlinear Analysis. Marcel-Dekker, New York (1999)

33. Ansari, QH, Yao, JC: A fixed point theorem and its applications to a system of variational inequalities. Bull Aust Math Soc. 59, 433-442 (1999). doi:10.1017/S0004972700033116

34. Alber, Ya: Generalized projection operators in Banach spaces: properties and application. Proceedinng of the Israe Seminar Ariel Israel Funct Differential Equation vol. 1, 1-21 (1994)

35. Alber, Ya: Metric and Genernalized Projection Operators in Banach Space: Properties and Application. In: Kartsatos, A (eds.) Theory and Applications of Nonlinear Operators of Accretive and Monotone Type. pp. 15-50. Marcel Dekker, New York (1996)

36. Ansari, QH, Lin, YC, Yao, JC: General KKM theorem with applications to minimax and variational inequalities. J Optim Theory Appl. 104(1):41-57 (2000)

37. Zeng, LC, Yao, JC: Existence theorems for variational inequalities in Banach spaces. J Optim Theory Appl. 132(2):321-337 (2007). doi:10.1007/s10957-006-9139-z

38. Zeidler, E: Nonlinear Functional Analysis and Its Application, I Fixed-Point Theorems. Springer, New York (1993)

39. Aubin, JP, Ekeland, I: Applied Nonlinear Analysis. Wiley, New York (1984)

doi:10.1186/1029-242X-2012-8

Cite this article as: Plubtieng and Thammathiwat: Existence of solutions of a new system of generalized variational inequalities in Banach spaces. Journal of Inequalities and Applications 2012 2012:8.

\section{Submit your manuscript to a SpringerOpen ${ }^{\circ}$ journal and benefit from:}

- Convenient online submission

- Rigorous peer review

- Immediate publication on acceptance

- Open access: articles freely available online

- High visibility within the field

- Retaining the copyright to your article

Submit your next manuscript at $\gg$ springeropen.com 